\title{
Improving Energy Estimation based Clustering with Energy Threshold for Wireless Sensor Networks
}

\author{
Gaurang Raval \\ CSE Department \\ Institute of Technology \\ Nirma University, Ahmedabad
}

\author{
Madhuri Bhavsar, Ph.D \\ CSE Department \\ Institute of Technology \\ Nirma University, Ahmedabad
}

\begin{abstract}
In this paper an energy usage estimation technique (LCEFCM) has been proposed which employs the Fuzzy C-Means clustering for creating clusters in the Wireless Sensor Networks. LCEFCM reduces the energy consumption considerably compared to other clustering methods like simulated annealing and K-Means clustering. It applies the dynamic clustering mechanism combined with balanced clustering method. LCEFCM outperforms LEACHC, LEACHC Estimate(LCE) and LCEKMeans for various performance measuring factors like network lifetime, data received, alive nodes etc.
\end{abstract}

\section{Keywords:}

WSN, Energy Estimation, Threshold, FCM

\section{INTRODUCTION}

Communication Technology foster the fast growing world with quick and reliable data transfer and information sharing. With the advent of various generations of networking technologies, applications have been developed for automatic data acquisition and information compilation and computation. Sensor networks is one such application where hundreds to thousands of sensor nodes are deployed for gathering information related to environment conditions, performing necessary simple computations and sharing the information with peer nodes or with an external Base Station. The main objective [1] of a wireless sensor network is to detect an event and dissipate the same to other nodes reliably. One of the issues in sensor networks is the limited energy availability to the remotely placed sensor nodes. These nodes are required to function with constrained resources [2] like less energy utilization with more desired lifetime. These nodes measure the environmental parameters and send back the results across the network to a base station where the data can be analyzed and eventually serve to initiate some action. Compared to all other operations a sensor node performs, communication is the costliest one. Major focus of the research work in sensor network is generally on finding ways to reduce radio transmission and reception counts. In-network data processing provides a means to reduce the number of wireless communications. Data aggregation is one of the mechanisms to perform In-network data processing. Data aggregation techniques can be an integral part of the routing process in the network. Various routing protocols exist which allow aggregation of data packets and routing happening together. Clustering approaches have become an emerging technology for building scalable, robust, energy-efficient WSN applications. Routing in WSNs can be generally categorized [3][4] into flat routing, hierarchical routing and location-based routing. In flat routing, every node has similar role to play. In hierarchical routing, however, nodes act differently as per the roles assigned to them. Clustering techniques enable formation of hierarchical structures in network for routing of necessary information to base station.

In cluster based data aggregation technique, every sensor node, associates itself with some cluster by communicating few small sized messages. One of the nodes in the group will act as a cluster-head $(\mathrm{CH})$ and sends the solicitation message across the network. The receiving members may join the group as per the proximity of that node with the sending node. The $\mathrm{CH}$ can determine the number of cluster members $(\mathrm{CMs})$ it can handle. A TDMA schedule is created for members to send data to $\mathrm{CH}$ and from there to BS. Once the cluster-head is elected, the data transmission from member node starts. All members will send data to $\mathrm{CH}$ in their designated TDMA slot. $\mathrm{CH}$ node can apply aggregation or summarization on the arrived data and forward it to the base station regularly till the end of the round. From next round onwards, the similar process of clustering and data collection continues as long as sufficient number of nodes are available to conduct this process.

In centralized version of clustering protocols, generally, the sensor nodes send their energy/location information to the BS. BS then applies suitable clustering technique to group the nodes in clusters and declares the list of $\mathrm{CHs}$ and $\mathrm{CMs}$. Afterwards, the $\mathrm{CHs}$ and CMs go through the steady state phase of data transmission. The reclustering process is called iteratively at every round. The centralized clustering protocol suffers from the excess energy usage during location or energy information transmission to BS. It severely affects the network lifetime if the base station is located far away from the deployment field. Various schemes have been proposed in the literature to reduce such transmissions to conserve the energy. In this paper, energy estimation based centralized clustering protocol is proposed. Simulation results bring out that the proposed approach improves lifetime of the network, with improved data delivery in the network. In section 2 related work is discussed in detail. Section 3 discusses the radio model and network assumptions. In section 4 proposed approach is described 
in detail. Section 5 presents evaluation of proposed approach with simulation results and section 6 concludes the paper.

\section{RELATED WORK}

Heinzelman [5] proposed the hierarchical routing protocol LEACH. Since its publication, it has been the defacto standard for hierarchical routing protocol design in wireless sensor networks. LEACH guarantees that the energy load is well distributed by dynamically created clusters electing cluster heads with optimal probability. Cluster head nodes summarize the data received from members. Then, it sends that aggregated data to BS. The cluster-head role is assigned on rotational basis during every round so that energy expenditure of being cluster-head is uniformly spread across the network. LEACH suffers from several drawbacks like non-uniform clusters and no residual energy considerations. Due to this, it does not create clusters uniformly over the deployment region. Sometimes, it may create clusters concentrated in a particular area of the deployment region leaving nodes uncovered.

In LEACH-C [6], all nodes send their energy and location information to the base station in every round. Then base station decides the cluster head and cluster members with the best possible grouping depending on the distance between cluster head and cluster members. Simulated annealing algorithm is applied to create the clusters. The nodes which are having suitable position such that the communication cost is minimum and their residual energy is above the average energy of network, are selected as cluster heads. The nodes are aware about whether they are cluster heads or cluster members, accordingly they schedule their transmissions using TDMA scheme. Cluster head aggregates the information arriving from member nodes to the base station at regular interval, at the end of the TDMA frame in a particular cluster. The process is repeated in every round during setup phase. Steady state phase is similar to that of LEACH protocol. Figure

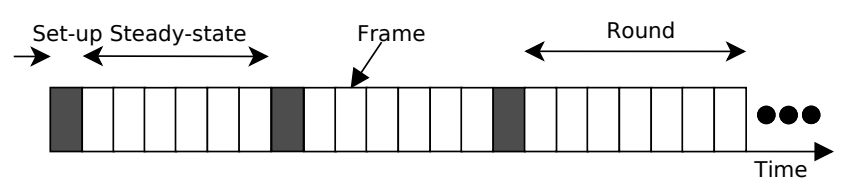

Fig. 1. Round of LEACH and LEACHC

1 shows how the LEACH or LEACHC protocol progresses with time. Initially it forms clusters during the setup phase and then after, steady-state phase starts. Every steady-state phase is assigned fixed time interval. In this interval, every cluster member sends data to its respective cluster-heads. Depending on the number of members present in every cluster, every member will get a TDMA slot at regular interval as shown in Figure 2 In [7] authors have proposed

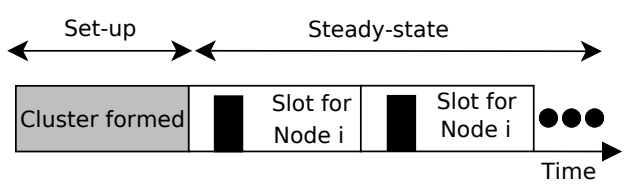

Fig. 2. Detailed Layout of LEACHC

K-Means based clustering where they minimized the objective function,

$$
F=\sum_{j=1}^{K} \sum_{i=1}^{N}\left\|x_{i}^{(j)}-c_{j}\right\|^{2}
$$

Here, $\left\|x_{i}^{(j)}-c_{j}\right\|^{2}$ indicates distance between a data point $x_{i}^{(j)}$ and centorid $c_{j}$. It indicates the distance of all data points from their respective centroids. In [8], authors have proposed balanced K-Means clustering algorithm wherein, they strictly assign fix number of members to the cluster during clustering process.

Fuzzy C-Means clustering algorithm is also being applied to various application domains by researchers. In [9] and [10], authors have used Fuzzy C-Means clustering algorithm for centralized clustering process. They try to optimize the objective function,

$$
J_{m}=\sum_{i=1}^{N} \sum_{j=1}^{C} \delta_{i j}^{m}\left\|x_{i}-c_{j}\right\|^{2}, \quad \text { where } \quad 1 \leq m<\infty
$$

where

$$
\delta_{i j}=\frac{1}{\sum_{k=1}^{C}\left(\frac{\left\|x_{i}-c_{j}\right\|}{\left\|x_{i}-c_{k}\right\|}\right)^{\frac{2}{m-1}}}
$$

The norm, $\left\|x_{i}-c_{j}\right\|$ measures the similarity (or closeness) of the data point $x_{i}$ to the center vector $c_{j}$ of cluster $j$. Cluster centers are computed with,

$$
C_{j}=\frac{\sum_{i=1}^{N} \delta_{i j}^{m} \cdot x_{i}}{\sum_{i=1}^{N} \delta_{i j}^{m}}
$$

The degree of membership for data point $i$ to cluster $j$ is initialized with a random value $\theta_{i j}, 0 \leq \theta_{i j} \leq 1$, such that $\sum_{j}^{C} \delta_{i j}=$ 1. The FCM algorithm refines the clustering process until the required minimum degree of membership is not achieved. The termination conditions are the least overlapping membership of members with neighboring clusters and the fuzziness coefficient of the membership. The terminating criteria can be specified as per the required accuaracy of the clustering process. Once the clusters are created with the centroids, the members near the centroid are labelled as cluster-heads depending on available energy of members.

In [11], authors have proved that energy estimation can be used to reduce the energy consumption with hardware specific component level energy modeling. Their estimations are close to the real measurements taken on the sensor hardware. They have applied node level energy estimations. In [12], authors have proposed linear energy model for node level energy estimations. Instead of collecting the raw residual energy data from individual nodes, in [13] authors apply in-network aggregation to generate composite residual energy scans. They apply network-level energy estimation method to reduce the energy consumption. In [14], authors have described network-level energy estimation for cluster based routing protocol. They have compared subtractive clustering with FCM based clustering. In [15], authors have analyzed the energy estimation comprehensively and compared their approach with other known LEACH variants. In [16], authors have proposed energy dissipation forecast and clustering management (EDFCM) protocol. EDFCM assumes the heterogeneous nodes in the deployment, accordingly applies the energy model to compute the energy drain out of the cluster head nodes and cluster members. 
It also applies optimal cluster head selection policy while electing cluster heads. In [17], LEACH-CCB protocol had been proposed where, $5 \%$ of alive nodes are elected as cluster heads in the network . The status message from member nodes is sent only once during the first round, during remaining rounds the energy is estimated with the known parameters like cluster heads, cluster member count, number of frames in the round etc. $10 \%$ of the nodes in the network are forced to go to sleep mode by BS, before clustering process. The nodes in sleep mode do not receive or transmit any data from neighbours or BS. Base station has complete control over the operation of all nodes. The base station chooses 5\% to be cluster heads, and the cluster heads of previous rounds are not eligible to participate in the cluster head selection unless, all nodes in the network have become cluster heads. This model randomly selects the nodes which are switching to sleep mode, which does not guarantee the availability of data from particular region even if it extends the lifetime. Also, the assumptions about initial energy and simulation results are not justified with respect to energy model applied. As 10\% nodes are in sleeping state, throughput should have been less by that factor but, results are not witnessing this fact. Also, authors have included the initial status message as part of throughput which seems irrelevant.

The energy required to send the energy status message is greater than the amount of energy needed to transmit the sensed data to the cluster head. Making use of the same centralized clustering scheme as in LEACH-C and reducing the number of communications between the nodes and base station, an energy efficient clustering scheme through estimate was proposed by Jim-Moo-Kim [18]. The protocol LEACH-CE achieves greater network lifetime than the previous protocols. The status message is received by the base station only at the setup phase of first two rounds. Third round onwards the remaining energy level of each node is calculated by the base station itself. The average energy expenditure of the cluster head and the node is calculated and subtracted respectively for the next round. The energy utilization of the nodes in LEACH-CE is optimal than in LEACH-C. The system lifetime, the live node count in the network at a given time, the useful data received by the base station is greater in LEACH-CE than LEACH-C. LEACHC-E [18] applies estimation of energy consumption during the clustering process. It collects energy and location information from nodes during first two rounds. Average energy usage by cluster-head and cluster members is derived after observing the energy usage during these two rounds. Later on, same is iteratively applied to subtract the energy consumption from cluster-heads or cluster members during the consecutive eight rounds. The frequency of energy information collection from members is shown in Figure 3 The results shown in Figure 5 for per round energy drain for

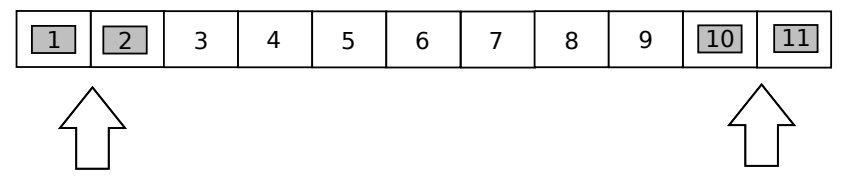

Fig. 3. Frequency of Energy Information Transmission [18

LEACHC and its variants suggest that, the estimated usage of energy consumption by members may not be accurate. As the clusters may be of different sizes, every node may be spending different amount of energy in a particular cluster. This parameter not only depends on the number of nodes present in the cluster, but also the distance of member with cluster head and distance of cluster-head with base station. During cluster formation in every round, cluster-heads also may be placed quite close to each other or they may be far from each other. Cluster-heads may be near the base-station or they may be away from the base station. LEACHC-E [18] uses estimated energy during $80 \%$ of the time during the network lifetime. Average energy usage analysis done during the first two rounds, may not be directly applied for $80 \%$ of the time.

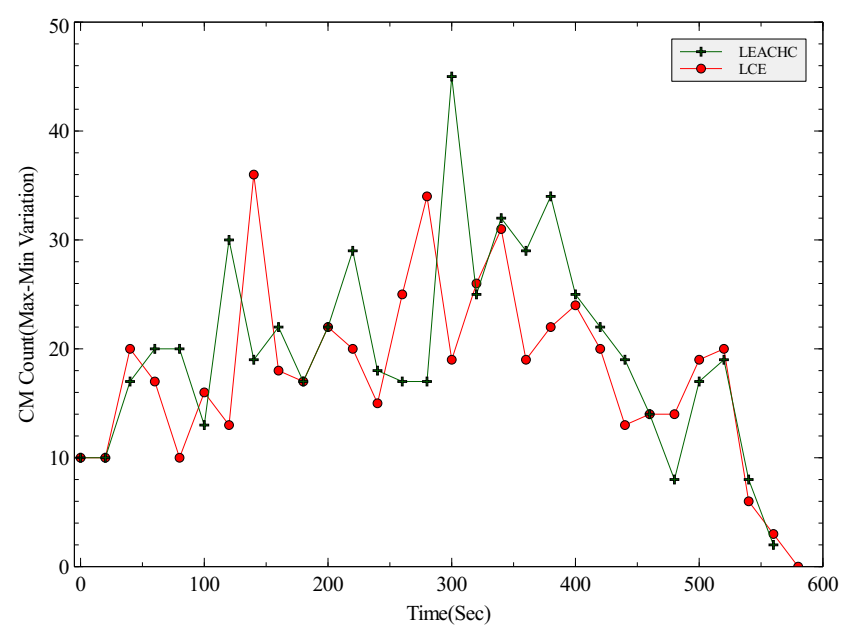

Fig. 4. Cluster Size Variations-LEACHC and LCE

Figure 4 shows the clustering effect of LEACHC and LEACHCE protocols. Both are showing large variation in minimum and maximum members being assigned to two distinct clusters, for the given cluster set at a particular round. As the clustering process shows large variation (Figure 4 in cluster members assignment to particular cluster, in every round, a cluster member will get different share of the total TDMA slots in the cluster. The placement of the nodes will also be different with respect to cluster-head. So using only two estimated energy usage parameters, one for cluster-head and another for cluster member for majority of the time during the progress of the algorithm does not seem appealing. The energy usage estimation needs a refinement here. Due to variations in the cluster sizes, the nodes expiry also may not be predicted with only two energy estimation parameters as used in LEACHC-E [18]. A node may expire little early than expected or may last longer than the estimated death time. This also depends on the number of TDMA slots assigned to that node during a round and also on the distance of the node with the cluster-head if it is cluster member; and with base station if it is cluster-head. Node death may be more accurately predicted with the integration of radio propagation model in the energy estimation calculations.

A threshold may be calculated considering the highest number of members being assigned to any cluster. The number of cluster members in any cluster in worst case can be made available after the careful analysis of the clustering process. The same worst case member count may be utilized to predict the node survival during a particular round. If the node can not survive the round as per the Likely to Die Threshold, $L D_{t h r}$, it will be not be made member of any cluster during a particular round. Consideration of such nodes during clustering will create clusters, wherein few members may die in the beginning of the round itself. So overall clustering process will be ineffective. To create the clusters where 


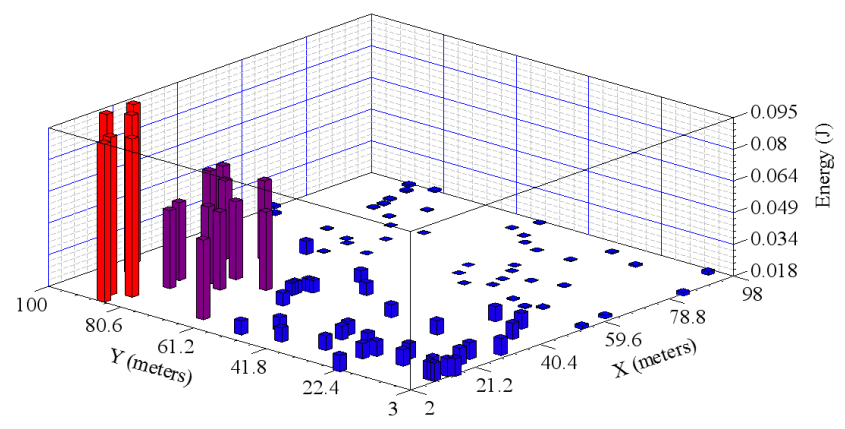

Fig. 5. Per Round Energy Drain - Worst Case

every member survives at least for that particular round, nodes with residual energy less than $L D_{t h r}$ should be avoided. Such TDMA slots will also be lost, as the nodes which have expired will not utilize these slots. These TDMA slots may be used by other nodes if the Likely to Die nodes are avoided from clustering process.

\section{NETWORK AND RADIO MODEL}

The energy model [6] used for simulating various protocols as found in literature is as shown in Figure 6 To transmit an L-bit

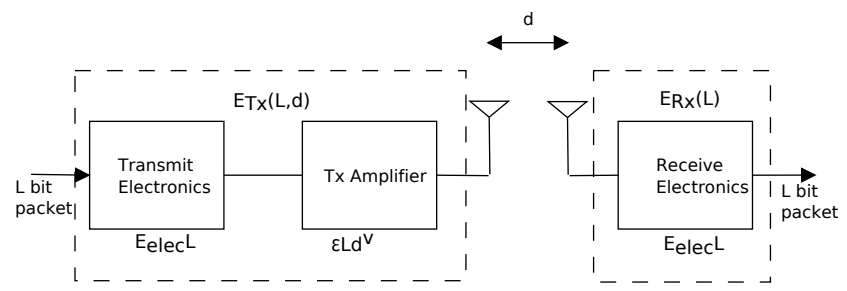

Fig. 6. Radio Energy dissipation model

message over a distance $d$, Energy $E_{T X}$ expended by radio is given by Equation 5 .

$$
E_{T X}(L, d)= \begin{cases}L\left(E_{\text {elec }}+\epsilon_{f s} d^{2}\right) & d<d_{0} \\ L\left(E_{\text {elec }}+\epsilon_{m p} d^{4}\right) & d \geq d_{0}\end{cases}
$$

Here,

$-E_{\text {elec }}$ is energy dissipated per bit to run transmitter and receiver circuit

$-\epsilon_{f s}$ and $\epsilon_{m p}$ depend on transmitter amplifier model

$-d$ is distance between sender and receiver. Crossover distance $d_{0}$ is $87 \mathrm{~m}$ as the cut-off point

Radio spends energy $E_{R X}$ in receiving an L-bit message as given by Equation 6 Energy required to receive $\mathrm{L}$ bits,

$$
E_{R X}(L)=L E_{\text {elec }}
$$

Here $d_{0}$ is the deciding factor whether to use free space propagation model or multi-path radio propagation model. $\epsilon_{f s}$ and $\epsilon_{m p}$ are the amplification components depending on the propagation model in use. Energy spent in transmitting the message is dependent highly on the distance between transmitter and receiver using free space model ( $d^{2}$ power loss) and multi-path fading ( $d^{4}$ power loss) channel models. In applications seeking small scale network deployment, typically in $100 \mathrm{~m} \mathrm{X} 100 \mathrm{~m}$ field, inter-node distance is greater than $d_{0}$, while in large scale network, inter-node distance is less than $d_{0}$.

\subsection{Assumptions}

During the simulations of the clustering protocols, following assumptions have been considered.

(1) Nodes are randomly deployed and they are stationary.

(2) Nodes are location-aware, which can be defined using localization methods or GPS.

(3) Each node has access only to information about its neighbors in one hop.

(4) All nodes have similar capabilities, processing, communication and initial energy.

(5) The transmission ranges of nodes are adjustable.

(6) Nodes are capable of making sleep and wake-up calls.

(7) All nodes can directly communicate with the static sink.

(8) Base Station is having sufficient amount of energy

\section{PROPOSED APPROACH}

The clustering process is done with FCM based clustering for the proposed approach to control the cluster size variation. Instead of estimating energy based on average observations, the $\mathrm{CH}$ and $\mathrm{CM}$ energy consumption is being estimated as follows.

\subsection{Set-up Phase Energy Usage}

Energy usage by $\mathrm{CH}$ node during setup phase can be represented as follows:

$$
E_{C H}=k E_{R x}+k E_{T x_{C M}}+k E_{T x_{B S}}
$$

where,

(1) $k E_{R x}$ represents the energy used by $\mathrm{CH}$ nodes to receive cluster information from BS

(2) $k E_{T x_{C M}}$ is the energy used by $\mathrm{CH}$ node to transmit the cluster information of that cluster to its Cluster Members

(3) $k E_{T x_{B S}}$ represents the energy spent by $\mathrm{CH}$ nodes for the transmission of the acknowledgment message for cluster information to BS

Energy usage by $\mathrm{CM}$ node during setup phase can be represented as follows:

$$
E_{C M}=(N-k) E_{R x}
$$

where,

(1) $(N-k)$ shows the count of cluster members

(2) $(N-k) E_{R x}$ is the amount of energy used by CM nodes to receive the $\mathrm{CH}$ announcements

\subsection{Steady-State Phase Energy Usage}

Energy usage by $\mathrm{CH}$ node during Steady State phase can be represented as follows:

$$
E_{C H}=\left(\frac{N}{k}-1\right) l E_{\text {elec }}^{R x}+\frac{N}{k} l E_{D A}+l E_{\text {elec }}^{T x}+l \varepsilon_{a m p} d_{\text {toBS }}^{n}
$$


Table 1. Radio parameters

\begin{tabular}{|l|l|c|}
\hline Operation & Symbol & Energy dissipated \\
\hline Energy consumed in electronics circuit for transmitting and receiving & $E_{e l e c}$ & $50 \mathrm{~nJ} / \mathrm{bit}$ \\
\hline Energy consumed by amplifier to transmit at shorter distance i.e. if $d_{t o B S}<d_{0}$ & $\epsilon_{f s}$ & $10 \mathrm{pJ} / \mathrm{bit} / \mathrm{m}^{2}$ \\
\hline Energy consumed by Amplifier to transmit at longer distance i.e. if $d_{t o B S} \geq d_{0}$ & $\epsilon_{m p}$ & $0.0013 \mathrm{pJ} / \mathrm{bit} / \mathrm{m}^{4}$ \\
\hline Energy consumed during data aggregation & $E_{D A}$ & $5 \mathrm{~nJ} / \mathrm{bit}$ \\
\hline
\end{tabular}

Energy usage by CM node during Steady State phase can be represented as follows:

$$
E_{C M}=l E_{\text {elec }}^{T x}+l \varepsilon_{a m p} d_{t o C H}^{n}
$$

Here, $E_{D A}$ is energy required to aggregate data, $d_{t o B S}$ and $d_{t o C H}$ is distance to base station and distance to cluster-head respectively. As discussed above, the $\mathrm{CH}$ and $\mathrm{CM}$ energy consumption is estimated by BS to predict the energy consumption for the current round using the available clustering information.

\subsection{Likely to Die Threshold}

To predict the node death, Likely to Die Threshold is calculated as follows,

$$
L D_{t h r}=\left(\frac{\text { Round Duration }}{\text { number of CMs } \times \text { slot-size }}\right) * E_{\text {Average }}^{C M t o C H}
$$

Average energy from cluster member to cluster-head is calculated as follows,

$$
E_{\text {Average }}^{\text {CMtoCH }}=\frac{E_{\min (C M t o C H)}+E_{\max (C M t o C H)}}{2}
$$

Round Duration is actually the time period fixed for each round. For example, if we take Round duration to be 20 seconds with slot size 2 seconds and 5 members in the cluster, then $L D_{t h r}$ for $X$ Joules of average energy $\left(E_{\text {Average }}^{C M t o C H}\right)$ can be calculated as,

$$
L D_{t h r}=\left(\frac{20 \text { seconds }}{5 \times 2 \text { seconds }}\right) \times X \text { Joule }=2 \times \text { XJoule }
$$

\subsection{FCM Clustering with Energy Estimation}

The clustering is done using Fuzzy C-Means clustering algorithm. The clusters are announced during the setup phase. During $10 \%$ of the time, energy information is directly received as per the strategy of Kim et al. [18]. Remaining $90 \%$ of the time, the energy usage of $\mathrm{CH}$ and $\mathrm{CM}$ is estimated as per the energy model based on radio propagation model. The actual energy received at every tenth round ensures that energy estimations are aligned with the actual residual energy of nodes and rectified accordingly. Algorithm 1 summarizes the proposed FCM based clustering approach with energy estimation.

\section{SIMULATION AND RESULT ANALYSIS}

Above mentioned protocols were implemented and simulated in NS-2 using $\mathrm{C}++$ and TCL. Table 1 shows the radio parameters used during simulations. Table 2 shows the simulation parameters used. The simulations were performed for various topologies ranging from 50 nodes to 200 nodes. The base station location was also varied, from center to $(50,175)$ in the deployment field. Figure 7 shows the controlled variation of clusters over time using FCM based clustering for 150 nodes deployment. As the clusters are not varying much in size, energy estimations can be more accurate and death of nodes can be predicted more accurately. As the FCM based

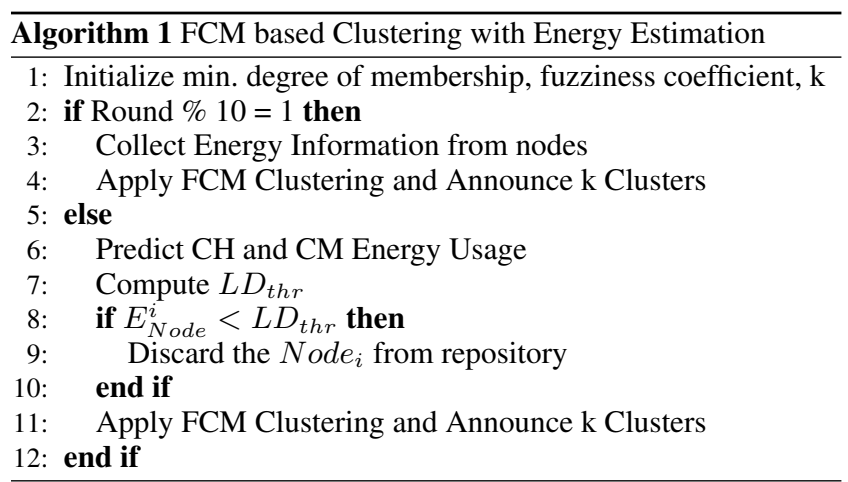

Table 2. Simulation Parameters

\begin{tabular}{|l|c|}
\hline Network Parameter & Value \\
\hline Node distribution & $(0,0)$ to $(200,200)$ \\
\hline BS location & Center and $(50,175)$ \\
\hline No. of Nodes & $50,100,150,200$ \\
\hline Initial Node Energy & $2 \mathrm{~J}$ \\
\hline Simulation Time & $3600 \mathrm{~s}$ \\
\hline Desired No. of cluster-heads & $5 \%$ \\
\hline Bandwidth of the channel & $1 \mathrm{Mbps}$ \\
\hline Packet header size & 25 Bytes \\
\hline Message size & 500 Bytes \\
\hline
\end{tabular}

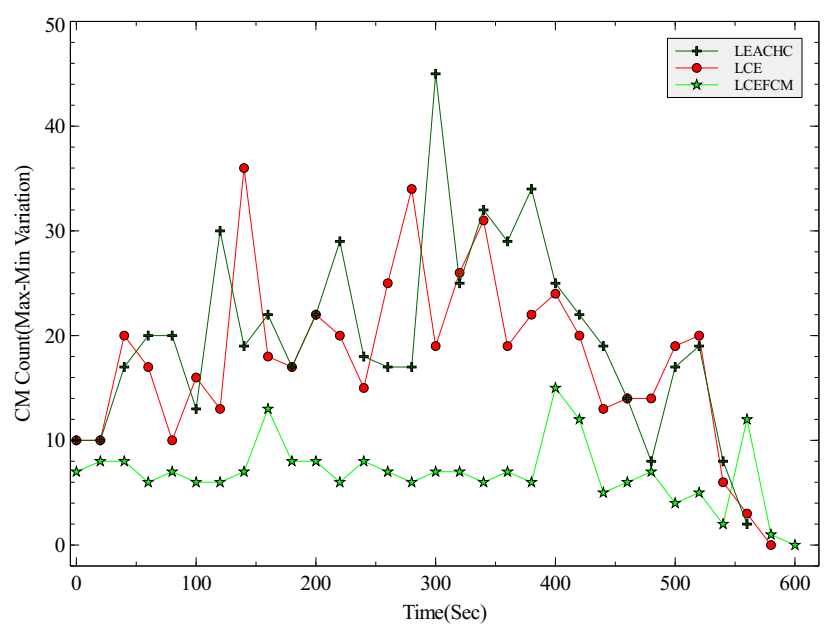

Fig. 7. Cluster Size Variations-LEACHC,LCE and LCEFCM 


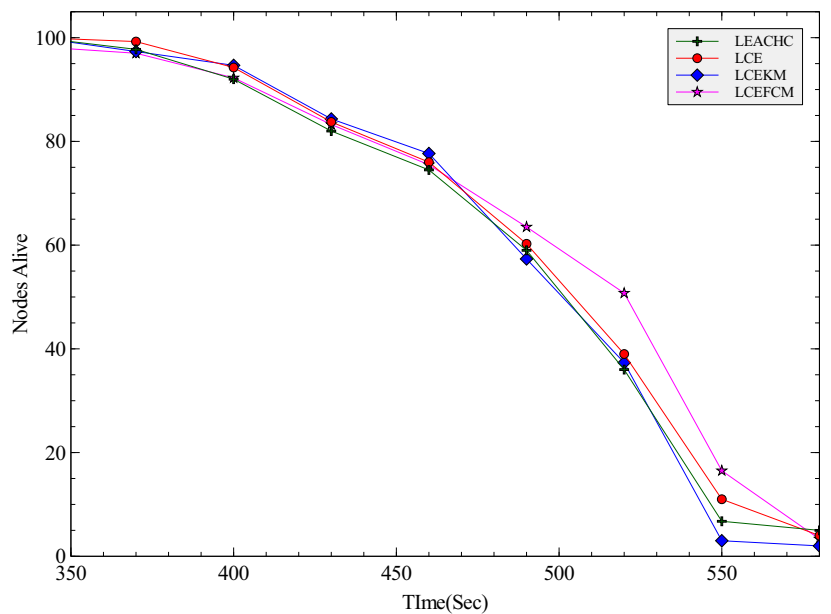

Fig. 8. Alive Nodes Comparison

clustering does better clustering compared to simulated annealing and K-Means, the improvement in the lifetime is also achieved. The same is evident in Figure 8 which shows alive nodes in the network for 100 nodes deployment. Also the improvement is due to the controlled cluster sizes and uniform energy drain from the network compared to non-uniform clusters. With better clustering

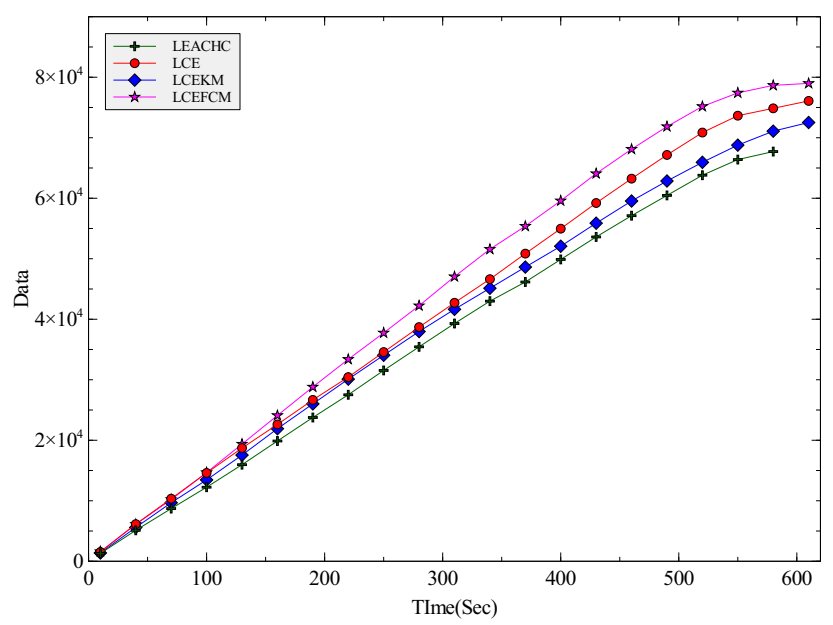

Fig. 9. Data Received at BS

achieved, the lifetime improves, thereby helping in delivering more amount of data compared to other protocols. The same may be observed in Figure 9 which shows the data received at BS for 200 nodes deployment. The cost of data transmission also comes down with controlled clustering with FCM based approach. This can be seen in Figure 10 which shows amount of data transmitted per unit energy for 200 nodes.

\section{CONCLUSION}

Energy estimations using absolute measures, like average energy usage by $\mathrm{CM}$ or $\mathrm{CH}$ for limited number of rounds does not work when clustering process is not generating quality clusters.

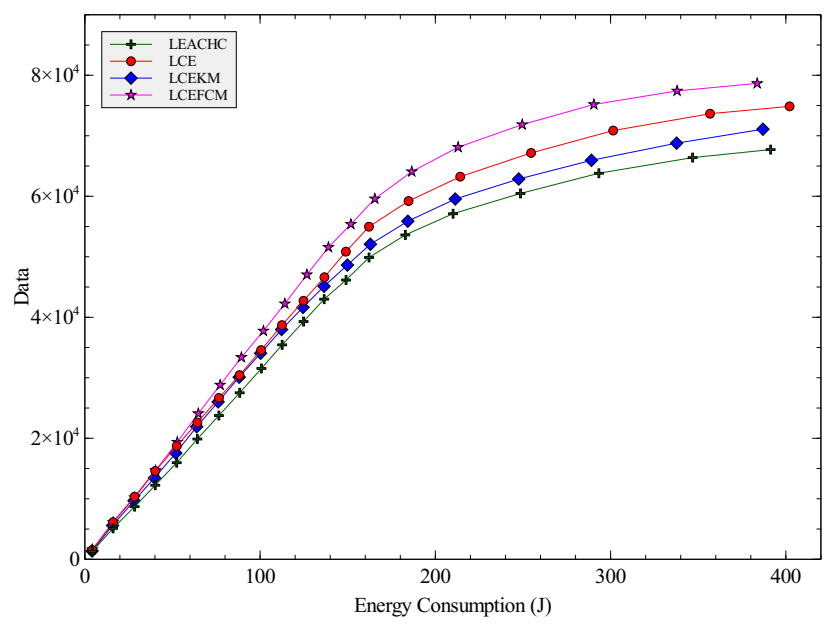

Fig. 10. Cost of Data Transmitted

When the cluster size is controlled during clustering process, energy estimations can be more accurate. This would also depend on the radio propagation effects. The lifetime and data delivery observed for the LCEFCM approach is higher than LCE, LCEKM and LEACHC. As the energy usage estimations for LCEFCM are more accurately computed compared to LCE, the death of nodes are more precisely calculated. This has an indirect effect on clustering process, as likely to die nodes are avoided from becoming members. The TDMA slots which would have been wasted otherwise, are now used by the live nodes. This results in an effective clustering, delivering additional amount of data in the same amount of time. So data delivery process is more effective. More data is delivered compared to LCE approach per unit time. The energy estimation based strategies are more efficient when the base station is placed far from the area to be monitored. The assumptions about radio propagation models have significant impact on the accuracy of energy estimations based protocols. Proposed approach can be further analyzed with various realistic radio propagation models. The network topology also plays important role when multiple nodes are communicating in clustered groups and directly with with the base station. For this paper, the topologies considered are uniform random topologies. The proposed approach can be also compared as an extension against several realistic sensor network topologies.

\section{References}

[1] D. Minoli K. Sohraby and T. Znati. Wireless Sensor Networks - Technology, Protocols, and Applications. John Wiley and Sons, 2007.

[2] Charles E. Perkins. Ad Hoc Networking. Addison-Wesley Professional, 1 edition, 2008. ISBN 0321579070, 9780321579072.

[3] K. Akkaya and M. Younis. A survey on routing protocols for wireless sensor networks. International Journal on Ad Hoc Networks, 3(3):325-349, 2005.

[4] Jamal N. Al-karaki and Ahmed E. Kamal. Routing techniques in wireless sensor networks: A survey. IEEE Wireless Communications, 11:6-28, 2004.

[5] Wendi Rabiner Heinzelman, Anantha Chandrakasan, and Hari Balakrishnan. Energy-efficient communication protocol 
for wireless microsensor networks. In System Sciences, 2000. Proceedings of the 33rd Annual Hawaii International Conference on, pages 10-pp. IEEE, 2000.

[6] Wendi B Heinzelman, Anantha P Chandrakasan, and Hari Balakrishnan. An application-specific protocol architecture for wireless microsensor networks. Wireless Communications, IEEE Transactions on, 1(4):660-670, 2002.

[7] P Sasikumar and Sibaram Khara. K-means clustering in wireless sensor networks. In Computational Intelligence and Communication Networks (CICN), 2012 Fourth International Conference on, pages 140-144. IEEE, 2012.

[8] Liansheng Tan, Yanlin Gong, and Gong Chen. A balanced parallel clustering protocol for wireless sensor networks using k-means techniques. In Sensor Technologies and Applications, 2008. SENSORCOMM'08. Second International Conference on, pages 300-305. IEEE, 2008.

[9] Ajay Singh Raghuvanshi, Sudarshan Tiwari, Rajeev Tripathi, and Nand Kishor. Optimal number of clusters in wireless sensor networks: a fcm approach. IJSNet, 12(1):16-24, 2012. doi: http://dx.doi.org/10.1504/IJSNET.2012.047707.

[10] DC Hoang, R Kumar, and SK Panda. Fuzzy c-means clustering protocol for wireless sensor networks. In Industrial Electronics (ISIE), 2010 IEEE International Symposium on, pages 3477-3482. IEEE, 2010.

[11] Adam Dunkels, Fredrik Osterlind, Nicolas Tsiftes, and Zhitao He. Software-based on-line energy estimation for sensor nodes. In Proceedings of the 4th workshop on Embedded networked sensors, pages 28-32. ACM, 2007.

[12] Ossama Younis and Sonia Fahmy. An experimental study of routing and data aggregation in sensor networks. In Mobile Adhoc and Sensor Systems Conference, 2005. IEEE International Conference on, pages 8-pp. IEEE, 2005.

[13] Yonggang Jerry Zhao, Ramesh Govindan, and Deborah Estrin. Residual energy scan for monitoring sensor networks. In Wireless Communications and Networking Conference, 2002. WCNC2002. 2002 IEEE, volume 1, pages 356-362. IEEE, 2002.

[14] Malka N Halgamuge, Siddeswara Mayura Guru, and Andrew Jennings. Energy efficient cluster formation in wireless sensor networks. In Telecommunications, 2003. ICT 2003. 10th International Conference on, volume 2, pages 1571-1576. IEEE, 2003.

[15] Malka N Halgamuge, Moshe Zukerman, Kotagiri Ramamohanarao, and Hai Le Vu. An estimation of sensor energy consumption. Progress In Electromagnetics Research B, 12:259-295, 2009. doi: 10.2528/PIERB08122303.

[16] Haibo Zhou, Yuanming Wu, Yanqi Hu, and Guangzhong Xie. A novel stable selection and reliable transmission protocol for clustered heterogeneous wireless sensor networks. Computer Communications, 33(15):1843-1849, 2010.

[17] SH Manjula, EB Reddy, K Shaila, L Nalini, KR Venugopal, and LM Patnaik. Base-station controlled clustering scheme in wireless sensor networks. In Wireless Days, 2008. WD'08. 1st IFIP, pages 1-5. IEEE, 2008.

[18] Jin-Mook Kim, Hyeon-Kyu Joo, Seong-Sik Hong, Woo-Hyun Ahn, and Hwang-Bin Ryou. An efficient clustering scheme through estimate in centralized hierarchical routing protocol. In Hybrid Information Technology, 2006. ICHIT'06. International Conference on, volume 2, pages 145-152. IEEE, 2006. 